\title{
An Optimized CNN based Real World Anomaly Detection in Surveillance Videos
}

\author{
Divya Thakur, Rajdeep Kaur
}

\begin{abstract}
Anomaly detection in automated surveillance video is an extremely monotonous process for monitoring for crowded scenes and surveillance videos are capable to incarcerate a mixture of sensible anomalies. An appropriate machine learning technique can help to train the Anomaly Detection System (ADS) in identifying anomalous activities during surveillance. To this end, we present an anomaly detection system that can be used as a tool for anomaly detection in surveillance videos using the concept of artificial intelligence. The main intention of the proposed anomaly detection system is to improve the detection time and accuracy by using the concept of Convolutional Neural Network (CNN) as artificial intelligence technique. In this paper we present a CNN based Anomaly Detection System (CNN-ADS), which is the combination of multiple layer of hidden unit with the optimized MSER feature by using Genetic Algorithm (GA). Here CNN is used for classifying the activity into normal and abnormal from the surveillance videos based on the fitness function of $G A$ which is used for the selection of optimal MSER feature sets. Further, Self adaptive genetic algorithm (SAGA) is adopted to efficiently solve optimization problems in the continuous search domain to select the best possible feature to segregate the pattern of normal and abnormal activities. The main contribution of this research is validation of proposed system for the large scale data and we introduce a new large-scale dataset of 128 hours of videos. Dataset consists of 1900 long and untrimmed real-world surveillance videos, with 13 sensible anomalies such as road accident, burglary, fighting, robbery, etc. as well as normal activities. The experimental results of the planned system show that our CNN-ADS for anomaly detection achieve essential improvement on anomaly detection presentation as compared to the state-of-the-art approaches. The dataset is available at: https://webpages.uncc.edu/cchen62/dataset.html. In this paper, to validate the proposed ADS we provide the comparison of existing results of several recent deep learning baselines on anomalous activity detection. The real-time ADS in surveillance video sequences using SAGA based CNN with MSER feature extraction technique is implemented using Image Processing Toolbox within Matlab Software.
\end{abstract}

Index Terms: Anomaly Detection System (ADS), Convolutional Neural Network (CNN), MSER Feature Extraction, Pattern recognition, Genetic Algorithm (GA).

\section{INTRODUCTION}

Reconnaissance is a fundamental piece of any country. CCTV cameras are pervasive and are utilized at different spots. A framework having the capacity to identify and report suspicious exercises is alluring and significant. In any case, an occasion that is irregular in one setting may not be suspicious in some other setting. Likewise, the inconsistencies are described by their properties in both the spatial just as the

Revised Manuscript Received on June 15, 2019.

Rajdeep Kaur, CSE Deptt., Chandiagrh University, Gharuan, INDIA.
Divya Thakur,CSE Deptt. , Chandigarh University, Gharuan, INDIA.

transient space. Thinking about the above difficulties, we look for an unsupervised methodology and proposed Anomaly Detection System (ADS). Human brain is the most intelligent organism, which leverages cognitive reasoning and processes visual data for high level semantic interpretation and gaining selective situational awareness. Over the previous few years, the computer visualization researchers have attempted similar capabilities to video analysis systems. With the accessibility of cheaper visual sensors, the need for understanding large quantity of video data is also increased. A significant application of video analysis is intelligent traffic surveillance system, which is used for forensic analysis or abnormal event detection/prediction. The ADS example is shown below figure 1 .

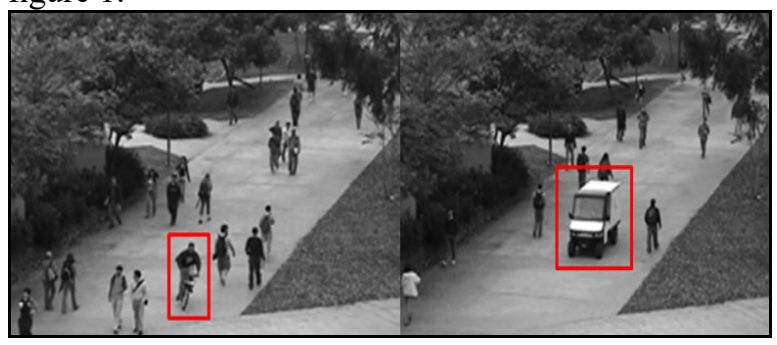

Figure 1: Anomaly Detection in Video

In the figure 1 , red color boundary is denoting the abnormal event which is different from the actual event. From the observation it is clear that all people are moving in actual event but some irrelevant event comes which is an abnormal event. The concept of Machine Learning (ML) approaches are popular in the area of video anomaly detection for automated learning and detection which is based on explicit or implicit model that enables classification of the patterns analyzed. In this paper we develops an activity recognition system which classify the abnormal/normal event from the surveillance videos using the Convolutional Neural Network (CNN) as ML approach with Genetic Algorithm (GA). The framework of proposed ADS is shown in the figure 2.

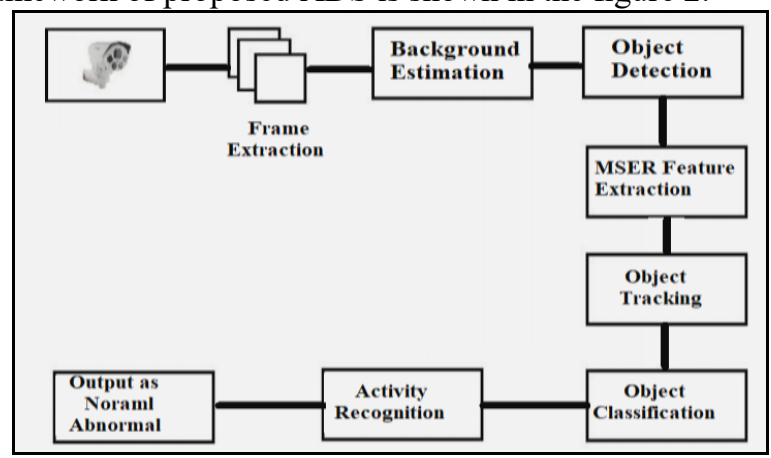

Figure 2:Framework of ADS 


\section{An Optimized CNN based Real World Anomaly Detection in Surveillance Videos}

Above figure describe the process of proposed ADS framework and the description of each blocks are given as:

Frame Extraction: It is the basic and primary step of any ADS. Frame extraction is a powerful mechanism that implements video content by selecting a set of summary frames to represent video sequences.

Background Estimation: Background estimation is the process of extracting, moving foreground objects from the stored background video frame or generated background frame form image series (video). Differentiating the moving objects from a surveillance video sequence is the principal and significant task in ADS and it is possible by the background estimation process.

Object Detection: Object detection is an innovative approach with computer vision and image processing that manages distinguishing examples of semantic objects of a specific class in digital image processing area. In the ADS, object detection is used as a Region of Interest (ROI) selection method using the concept o image segmentation. The most important objective of the object detection in ADS is to partition a video frame into commonly exclusive and exhausted regions such that each ROI is spatially contiguous and the pixels within the region are homogeneous with respect to a pre defined criteria.

MSER Feature Extraction: Extract feature from the ROI of video frame is called feature extraction algorithm and in this work we use MSER feature extraction technique. MSER is a method for blob detection in video frames. The MSER algorithm extracts feature from a video frame in terms of number of co-variant regions, called MSER feature. An MSER feature is a stable connected component of some gray-level sets of the video frames.

Object Tracking: It is the approach toward discover a moving object after some time utilizing a video. Object tracking is a task within the era of computer vision that consists on the extraction of the motion of an object from a sequence of video frames estimating its trajectory. In ADS, object tracking is used to analyze the behavior of moving objects with respect to the time.

Object Classification: It is a method to classify the tracked object using the concept of ML. SAGA based CNN is used in the proposed ADS as a object classifier to classify the object as normal or abnormal activity with the help of GA as an optimization technique.

Motivation and contributions: The purpose of video content analysis is to find consequential structures and samples from video data. The assignment of activity detection is to overpass the gap among the numerical pixel level data and a high-level abstract activity account. Anomaly detection in video surveillance is a demanding and challenging assignment due to many complicated problems, such as low camera quality, high noise, illumination change and deformation in the scenes and interaction between multiple events. From these types of challenging task we present an optimized CNN based real world anomaly detection in surveillance videos. In simple words, this paper makes the following contributions.

* We propose a new temporal segmentation technique based on the thresholding method according to the events of video.

* To classify the normal an abnormal event in surveillance video, CNN technique is used with segmented frames of video with optimized feature using GA.
* We design a novel objective function for GA to optimize the MSER feature of segmented ROI of video frames.

* For the validation of proposed ADS, we evaluate performance parameters of proposed work like error rate and accuracy rate and compare with state-of-the-art approaches.

This paper presents an optimized $\mathrm{CNN}$ based real world anomaly detection in surveillance videos and their comparison with existing trends. Specifically, in section 2, we present the literate survey (background survey) of existing work for anomaly detection in surveillance videos. The architecture of proposed work is described in the section 3. The simulation result is cover in section 4 and we conclude with discussions on current challenges and future trends in section 5 .

\section{Background Survey}

In this section, we analyze the survey of existing work based on different issues in anomaly detection system in surveillance videos using different techniques. Waqas Sultani in 2018 [1] proposed a model of Real-world Anomaly Detection in Surveillance Videos. They proposed to learn anomalies by exploiting both quality and weak quality videos. Due to lack of annotating the anomalous segments or clips in training videos, which is very time consuming, they planned to learn anomaly through the deep multiple instance ranking framework by leveraging weakly labeled training videos, i.e. the training labels (anomalous or normal) are at video- level instead of clip-level. In this approach, they consider normal and anomalous videos as bags and video segments as instances in multiple instances learning (MIL), and automatically study a deep anomaly ranking model that predicts high anomaly score for anomalous video segments. Due to use the only machine learning technique, the classification accuracy very low and need to improvement by integrating the optimization technique along with the classifiers. Shih-Chung Hsu in 2018 [2] planned video-based abnormal human behavior detection for psychiatric patient monitoring. They proposed an unsupervised learning using the N-cut algorithm along with the SVM to label the video segments and then apply the Condition random field (CRF) with an adaptive threshold to distinguish the normal and abnormal events. The main aim of hybridization of $\mathrm{N}$-cut with SVM is to enhance the number of control commands, get better detection accuracy and minimize the detection time. The experimental results show its competitive performance for anomaly event detection in video surveillance and there is a lot of scope to improve the detection accuracy using optimization. Shaonian Huang in 2018 [3] presents learning multimodal deep representations for crowd anomaly event detection. Low-level visual options, energy options, and motion map options area unit at the same time etracted supported spatiotemporal energy measurements. Three convolutional restricted Boltzmann machines area unit trained to model the mid-level feature illustration 
of traditional patterns. Then a multimodal fusion theme is employed to find out the deep representation of crowd patterns. Supported the learned deep representation, a one-class support vector machine model is employed to absorb anomaly events. The proected technique is evaluated using two obtainable public datasets and compared with progressive ways. The experimental results show its competitive performance for anomaly event detection in video surveillance and there is a lot of scope to improve the detection accuracy using optimization. Kai-Wen Cheng in 2015 [4] presented a hierarchical framework for local and global anomaly detection. They rely on a bottom-up greedy algorithm and GPR to cluster, learn, and infer the semantic (appearance) and structural (position) relationship of the nearby STIPs. A consistent detection scheme is also presented. The new method can achieve at least $85 \%$ detection rate based on the four challenging datasets and provide competing performance against the previous works while maintaining much lower space-time complexity as only the sparse STIPS are dealt with. They can't achieve more than $90 \%$ of detection rate and there is any types of feature optimization technique are not used so the anomaly retrieval process becomes difficult. Cheng in 2015 [5] proposed hierarchical framework for local and global anomalies detection. They rely on a greedy method and Gaussian process regression to cluster, learn, and infer the semantic (appearance) and structural (position) relationships of the nearby STIPs. Their method achieves at least $80 \%$ detection rate based on the three challenging datasets and provides competing performance compared with previous works that characterize the relationships of densely-sampled patches while maintaining much lower space and time complexity but, they can't attain a good detection rate and the use of GPR clustering as segmentation is a random process which affects the detection results. Vartika Agrawal in 2015 [5] presented a comparative study of image segmentation and classification over CT pictures of cervical cancer. For image segmentation results obtained from Otsu segmentation isn't adequate for additional process however ACM has provided satisfying result. Therefore it can be concluded that ACM is suitable segmentation technique for CT pictures of cervical cancer. Moreover, image classification is performed by choosing options with the assistance of Artificial Bee Colony algorithm, classifier applied is k-NN and SVM. K-NN is showing 97\% accuracy with biased dataset and 100\% accuracy with unbiased dataset. While SVM with linear kernel is showing 93\% accuracy with biased data set and 99\% accuracy when SVM with radial basis as a kernel is applied on frame data. So it is finished that SVM with radial basis operate as kernel is best for classification, however biasness in data set can have an affect on the result.

Based on the survey we conclude some important point which helps to short out existing problem. Our contributions in this paper to solve above mention problems are presented in three fold. Firstly, we introduce a completely automated hybrid method for background segmentation and object detection by using threshold based approach. To the best of our awareness, our proposed work is among the first few attempts to use the concept of thresholding based background segmentation to tackle this challenging problem. Secondly, we design an appropriate feature selection algorithm with the help of GA with a novel fitness function. Our results show that this fitness

function can further improve the performance by removing the unwanted feature from the extracted feature set. So the uniqueness of extracted feature is more for normal and abnormal data. At end, we comprehensively evaluate the effectiveness, efficiency and the generalization capability of the proposed model with UCF-Crime Dataset using CNN as a classifier. Our model can be easily generalized to other challenging anomaly detection problems and also improve the accuracy of detection.

\section{Structure of Proposed Methodology}

The proposed an optimized CNN based real world anomaly detection in surveillance videos consists of four main steps. Firstly, extraction of frames from the surveillance video is applied for the further processing. After that, various image pre-processing techniques is applied to improve image quality and suitable segmentation technique will be applied to separate out background from the frame to extract exact Region of Interest (ROI). Here, to enhance uniqueness of extracted feature using MSER, Genetic Algorithm is used along with the novel fitness function to minimize the feature selection problem. Finally, recognition will be performed using CNN model to produce desired output for the automatic intelligent anomaly detection model

\begin{tabular}{|c|c|c|c|c|}
\hline Fra & Pre-pr & MSE & Featu & $\mathrm{CN}$ \\
\hline me & ocessi & $\mathrm{R}$ & re & $\mathrm{N}$ \\
\hline Extr & ng on & Featur & Opti & for \\
\hline actio & extract & e & mizat & Ano \\
\hline $\mathrm{n}$ & ed & Extrac & ion & maly \\
\hline from & frames & tion & Usin & Dete \\
\hline Vide & (ROI) & from & g GA & ction \\
\hline
\end{tabular}

Figure 3: Block diagram of proposed ADS

The challenge of this research work is to detect the anomaly in surveillance video and train the system using $\mathrm{CNN}$ on the basis of the features extracted from the ROI of lesion using MSER descriptor. After the feature extraction, feature selection is performed by using GA and the block diagram of proposed ADS is show in the figure 3 . The subsequent steps demonstrate the variety of phases that need to be accomplished.

Frame Extraction as Data Acquisition: Upload the surveillance video from the database of different classes to train and test the system. In both section of ADS, training as well as testing video is uploaded for processing. In training the number of video is more but in testing single test video is uploaded. The frame extraction process is known as Frame Extraction as Data Acquisition (FEDA) and the algorithm of FEDA is given as:

\section{Algorithm 1: FEDA Algorithm}

Input: Video Address $\rightarrow$ Full path of Video

Output: Extracted Frames

1. Define video browsing option for the video uploading

2. Pathname $=$ Browse (Video format (x.avi), Title of uploading)
3. Video = Video

Reader(Pathname) 


\author{
4. Num $=$ Video. Number of Frames \\ 5. for $\mathrm{i} \rightarrow$ Num \\ 6. Full-path $=($ Pathname with Filename $)$ \\ 7. $\quad$ Frames (i) $=$ Read (Full-path, i) \\ 8. End \\ 9. Return: Frames as Extracted Frames \\ 10. End
}

Pre-processing: Pre-Processing is done to remove various type of noise that are inherited in the frames to enhance the quality of extracted frames in proposed ADS. In every recognition system, selection of popper ROI is the major factor and need to remove extra part form the data. In pre-processing after that, threshold based segmentation is used to find out the better region of interest (ROI) which helps to achieve better detection accuracy. For the selection of ROI from extracted frames, threshold based segmentation is used which is depend on the morphological operations like binarization, thinning, etc. Morphological operation is a collection of non-linear operations related to the shape or morphology of features in an image. Apply Morphological operations on the binary image to find out the exact region of lesion within the image using the some basic operations. There are a lots of morphological operations are available but used operations are given in the below figure.

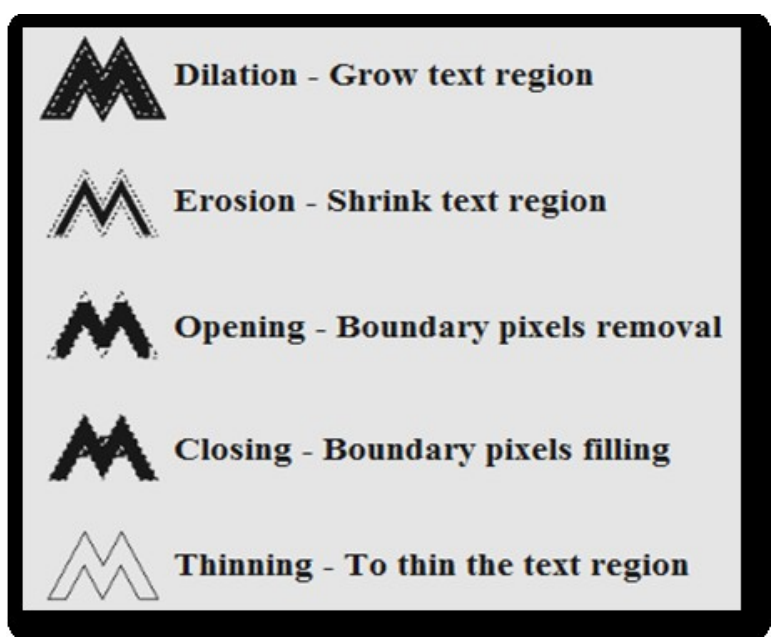

Figure 4: Morphological Operations

Above figure4 represents the morphological operations which are used in the proposed ADS. In the proposed work, morphological operations help to find out the exact region of objects and separate the extra region from images. The used pre-processing steps are:

1. Color Conversion:Color conversion is applied on the extracted frame from the video to convert into single band (Gray Image) which helps in the minutiae extraction.

$$
\text { Graylmage }=0.299 \times R_{b}+0.587 \times G_{b}+0.114 \times B_{b}
$$

Where, $R_{b}$ : Red band of an image

$\mathrm{G}_{\mathrm{b}}$ : Green band of an image

$\mathrm{B}_{\mathrm{b}}$ : Blue band of an image

2. Binarization: The binarization process is carried out using the following equation with threshold value.

$$
\operatorname{Bimg}(i, j)=\left\{\begin{array}{lr}
1 & \text { ifFimg }(i, j) \geq \text { Threshold } \\
0 & \text { else }
\end{array} .\right.
$$

As shown in the equation (2), Bimg is binary image and Fimg is frameof video with row (i) and columns (j). The binarization algorithm of proposed work is given as:

\section{Algorithm 2:Binarization Algorithm}

Input:Fimg $\rightarrow$ Frame of Video

Output:Bimg $\rightarrow$ Binary Image

1.Define Row (R) and Columns (C) of Fimg

2. For $i=1 \rightarrow R$

3. For $j=1 \rightarrow C$

4. Using equation (4)

5. If Fimg(i, j) $>$ Average (Fimg)

6. $\operatorname{Bimg}(i, j)=1$

7. Else if Fimg(i, j) < Average (Fimg)

8. $\operatorname{Bimg}(i, j)=0$

9. End

10. End

11. End

12. Return: Bimg as a binary image

13. End

Area Opening: The area opening is performed to remove the pixels from the boundary of object region in image so we can find out the well appropriate region of moving object. The opening is performed by using the given equation:

OpenImage $=\left\{\begin{array}{l}0, \text { Pixels }<\text { Threshold } \\ 1, \text { Pixels } \geq \text { Threshold }\end{array}\right.$

Where, Pixels : Binary image pixels

Threshold: Threshold pixels value which helps to remove the boundary pixels

On the basis for morphological operations, Threshold based ROI segmentation algorithm is designed and the algorithm of Optimized K-means is given as:

\section{Algorithm 3: Threshold based ROI segmentation}

Input: Bimg $\rightarrow$ Binary Image

Output: $\mathrm{ROI} \rightarrow$ Object ROI from frames

1. $[\mathrm{R}, \mathrm{C}, \mathrm{P}]=$ size $(\mathrm{Bimg})$

2. Threshold $_{\text {value }}=50 \%$ of $\max$ value

3. Threshold $=$ Threshold value

4.MaskImg =Morphological (Bimg, Threshold)

5. Boundaries $=$ bwboundaries (MaskImg)

6.Segmented Region $=$ Boundaries

7. fori $\rightarrow$ 1: $P$

8. Segmented Image $=$ BimgX Segmented Region

9. End

10. Return; Segmented Image as ROI of extracted frames 11. End

Feature Extraction: Extract feature from the ROI of frames based on the MSER feature extraction algorithm. After the feature extraction algorithm, a set of feature is return by the MSER algorithm in terms of feature points. The MSER algorithm is given as:

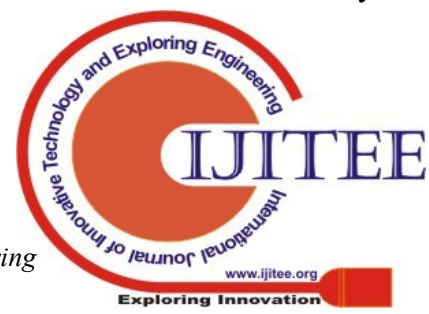




\begin{tabular}{l}
\hline Algorithm 4: MSER Algorithm \\
\hline Input: ROI $\rightarrow$ ROI image \\
Output:MSER_Points $\rightarrow$ Feature points \\
1.Load ROI data of extracted frames \\
2.Calculate size of dataset [Row, Col.] \\
3. fori $\rightarrow \mathbf{1}$ to all Row \\
4. for $\mathbf{j} \rightarrow \mathbf{1}$ to all Col \\
5. Local Intensity=intensity (ROI $(\mathrm{i}, \mathrm{j})$ ) \\
6. Connected Component=Component (Local Intensity $(\mathrm{i}, \mathrm{j})$, \\
8) \\
7. Threshold =Stable (Connected Components) \\
8.MSER_Points=Filtering (Threshold == True) \\
9. End \\
10. End \\
11. Return: Feature_descriptor as Fpoints \\
12. End
\end{tabular}

After the feature extraction algorithm applied on the ROI, we obtained below given results which are useful in training as well as classification process of proposed ADS. To extract feature from extracted ROI,MSER Descriptor is used and applied on the ROI. After the algorithm is applied on the ROI of extracted frames, the marked image with MSER point is given in the figure 5

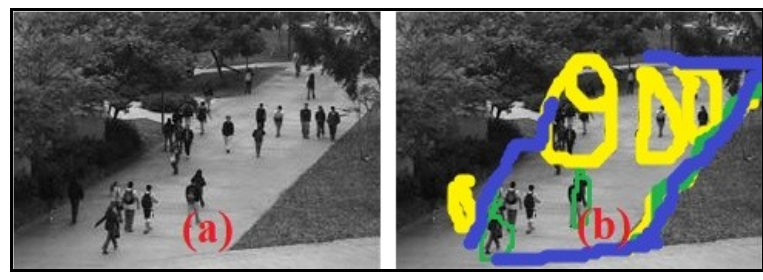

Figure 5: MSER Feature of Extracted ROI

Figure 5 represents the MSER region which count as MSER feature using the MSER descriptor. In the figure, (a) is the original image and (b) is representthe MSER region of original image which is considered as a set of MSER feature. Feature Optimization: For the best and optimal feature selection from the extracted MSER feature set, genetic algorithm is used as feature optimization technique and the algorithm of GA is give as :

\begin{tabular}{l} 
Algorithm 5: Genetic Algorithm \\
\hline Input: MSER_Points $\rightarrow$ Feature points \\
Output: Optimized MSER_Points $\rightarrow$ Optimized Feature \\
points \\
1. Initialize GA in within the ADS \\
2. Define population size, selection function, mutation \\
function, crossover function etc (Default). \\
3. Data $=$ MSER Feature Set \\
4. $\mathrm{F}_{\mathrm{s}}=$ Data value one by one \\
5. $\mathrm{F}_{\mathrm{t}}=$ Threshold value from the Data (Average of Data) \\
6. Define fitness function of GA using give equation (4) \\
$\quad \quad$ Fitness Function $=\left\{\begin{array}{l}f s \\
f t\end{array}\right.$ if ... (4) \\
7. No. of variables $=1$ \\
8. for i $\rightarrow \mathbf{1}$ to Data \\
9. Optimal Feature $=$ GA (Fitness_function, Initialize GA, \\
No. of variables)
\end{tabular}
10. End
11. Return: Optimal Feature as an Optimized MSER_Points 12. End

Training: Initialize $\mathrm{CNN}$ for classification purpose using two phases, namely, training and testing. After the training of system, we save the trained structure which is use in the classification section to classify the anomaly from surveillance video. In the testing phase, the test video is uploaded and repeats the all steps. In the classification section, test video MSER feature is matched with trained $\mathrm{CNN}$ structure and return results type as normal and abnormal events. CNN has inbuilt feature extraction method but when we pass input to CNN as MSER feature the accuracy of system is improved and the used CNN algorithm is given as:

\begin{tabular}{l} 
Algorithm 6: CNN Algorithm \\
Input: Optimized MSER_Points $\rightarrow$ Optimized feature points \\
as training data (T), Target $(\mathrm{G})$ and Neurons $(\mathrm{N})$ \\
Output: Type of event as normal and abnormal \\
Initialize CNN with parameters \\
- Epochs (E) \\
- Neurons (N) \\
- Performance parameters: Cross Entropy, Gradient, \\
Mutation and Validation \\
$-\quad$ Training Techniques: Scaled Conjugate Gradient \\
(Trainscg) \\
- Data Division: Random \\
For each set of T \\
If Training Data $\varepsilon$ Normal \\
$\quad$ Group (1) = Training data normal \\
Else if Training Data $\varepsilon$ Abnormal \\
$\quad$ Group (2) = Training data of abnormal \\
Else \\
$\quad$ Group (3) = Extra \\
End \\
Initialized the CNN using Training data and Group \\
Net = patternnet (N) \\
Set the training parameters according to the requirements and \\
train the system \\
Net = Train (Net, Trainingdata, Group) \\
Classification Results = simulate (Net, Test Data Optimized \\
Feature) \\
If Classification Results = True \\
Show classified results in terms of the normal and abnormal \\
events \\
Calculate the performance parameters \\
End \\
Return: Classified Results \\
End \\
\hline
\end{tabular}

UCF-Crime Dataset: Figure 6 represents the samples of used dataset in proposed ADS using a CNN along with GA based on the hybridization. In the database all videos are surveillance video with avi format and in the dataset 


\section{An Optimized CNN based Real World Anomaly Detection in Surveillance Videos}

videos any type of compression is not applied. All images are non-compressed and electronic noise free. In the dataset, mainly two types of categories of videos are presents, first is normal and another is abnormal. Dataset is a new large-scale dataset, called UCF-Crime, to evaluate our method. It consists of long untrimmed surveillance videos which cover 13 real world anomalies, including Abuse, Arrest, Arson Assault, Road Accident, Burglary, Explosion, Fighting, Robbery, Shooting, Stealing, Shoplifting, and Vandalism. These anomalies are selected because they have a significant impact on public safety
Figure 6: Dataset Sample of Proposed Work

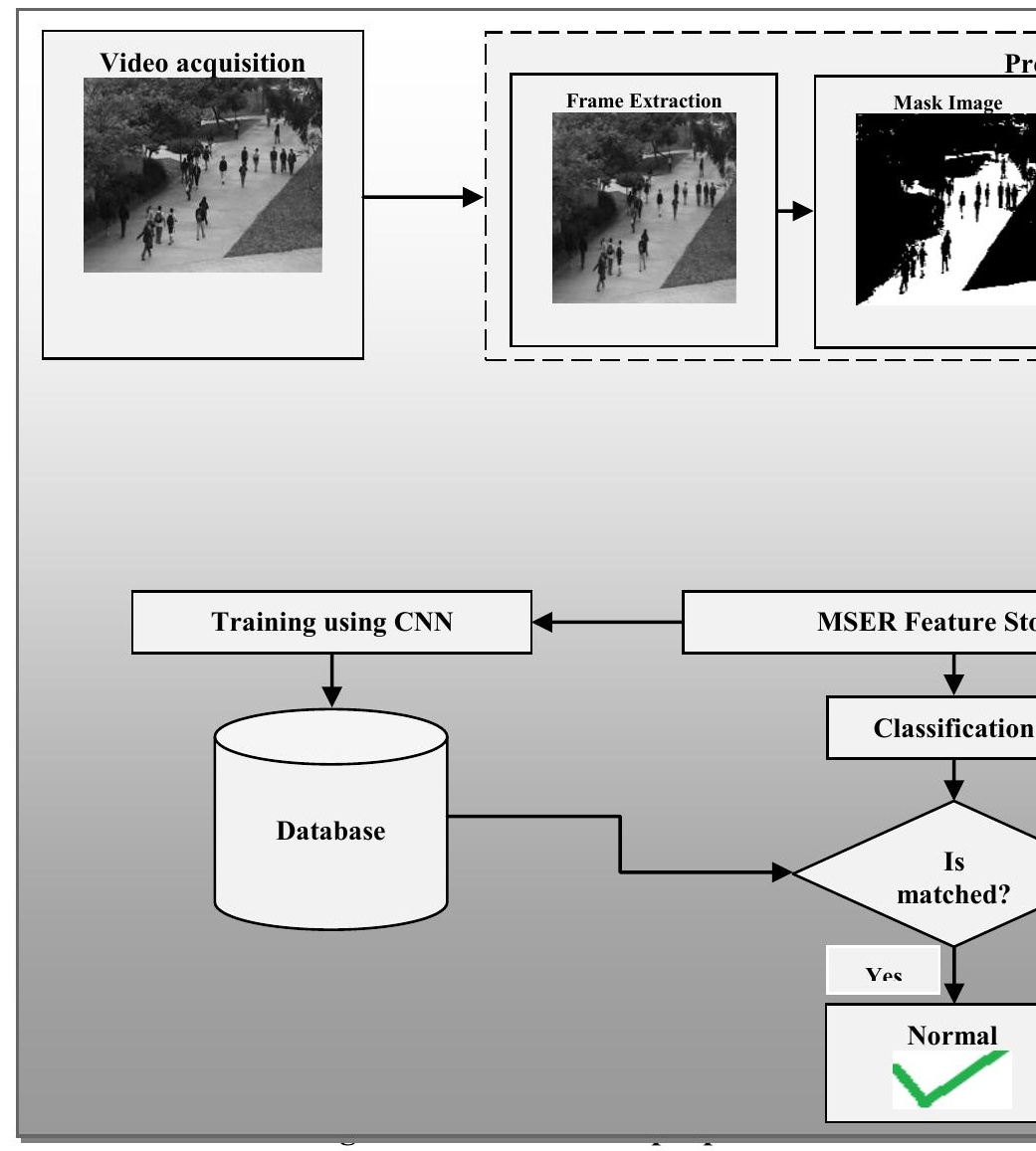

The flow chart of the proposed ADS is shown in figure 7 with training and testing process with surveillance videos and implementation steps are:

Step 1: Upload the video for simulation of proposed video ADS.

Step 2: Apply pre-processing on uploaded video in both sections training as well as testing to extract the frames from video for further processing.

Step 3: Develop a code for the segmentation of background and foreground from the pre-processed data in training as well as testing section.

Step 4: Apply MSER for feature extraction process from the segmented region of video frames.

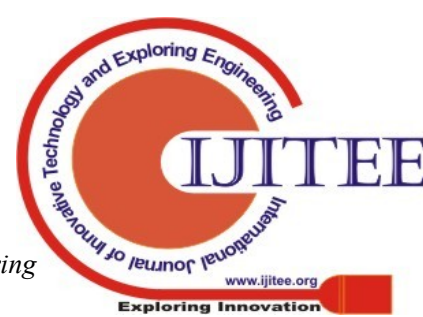


Step 5: Apply GA on extracted feature to optimize the feature sets and create a set of unique feature for anomalous and non anomalous data

Step 6: After that in the classification section, classification of events occurs according to the trained CNN structure and the performance parameters of ADS like Error rate and Accuracy rate is calculated.

\section{Results and Discussion}

In this section, the simulation result of proposed ADS is discussed and the efficiency of proposed work is compared with existing work [1]. The training and testing of the proposed mechanism is evaluated by UCF-Crime Dataset. By adapting the established proposed algorithms, below outcomes are computed with quality based parameters, such as Error and accuracy. a comparison is drawn with the existing work 1 to shown the effectiveness of the proposed work with respect to the normal and abnormal events based on the three sample data from each categories and fot the graphic on the three sample data from each categories and for the graphical representation we calculate their average value like Average Error Rate, and Average Accuracy of system.

\section{TABLE L: Test results of proposed method}

\begin{tabular}{|c|c|c|c|}
\hline Erent Types & Test Sample & Error Rate & Accuracy \\
\hline \multirow{3}{*}{ Sormal } & Test Sample 1 & 1.983 & 98.017 \\
\cline { 2 - 4 } & Test Sample 2 & 1.979 & 98.021 \\
\cline { 2 - 4 } & Test Sample 3 & 0.986 & 99.014 \\
\hline \multirow{3}{*}{ Abnormal } & Test Sample 1 & 0.937 & 99.063 \\
\cline { 2 - 4 } & Test Sample 2 & 1.963 & 98.037 \\
\cline { 2 - 4 } & Test Sample 3 & 0.982 & 99.018 \\
\hline
\end{tabular}

\begin{tabular}{|c|c|c|c|}
\hline Erent Tppes & Iets Sample & Error Rate & $\mathrm{AIC}$ \\
\hline \multirow[t]{3}{*}{ Tormal } & Test Sample I & 4.914 & 95.086 \\
\hline & Test Sample? & 6.992 & 93.008 \\
\hline & Test Sample\} & 2.899 & 97.101 \\
\hline \multirow[t]{3}{*}{ Abnormal } & Test Sample! & 6.998 & 93.102 \\
\hline & Test Sample? & 6.994 & 93.106 \\
\hline & Test Sample\} & 3.819 & 96.181 \\
\hline
\end{tabular}

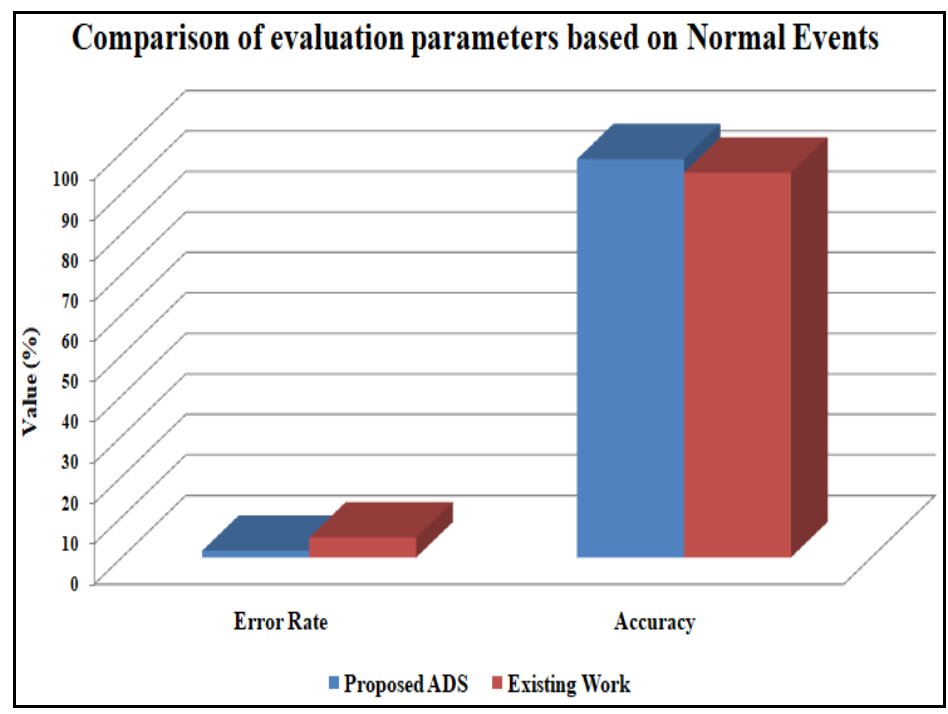

Figure 8: Comparison of evaluation parameters based on Normal Events

The comparison of evaluation parameters for proposed and existing work is depicted in figure 8. For the proposed work on the basis of normal event data, average error rate is $1.64 \%$ and average accuracy (AUC) $98.36 \%$. By using the concept of GA with MSER and CNN, the accuracy of proposed work is improved because the average error rate is $4.94 \%$ and average accuracy (AUC) $95.06 \%$ for the existing work.

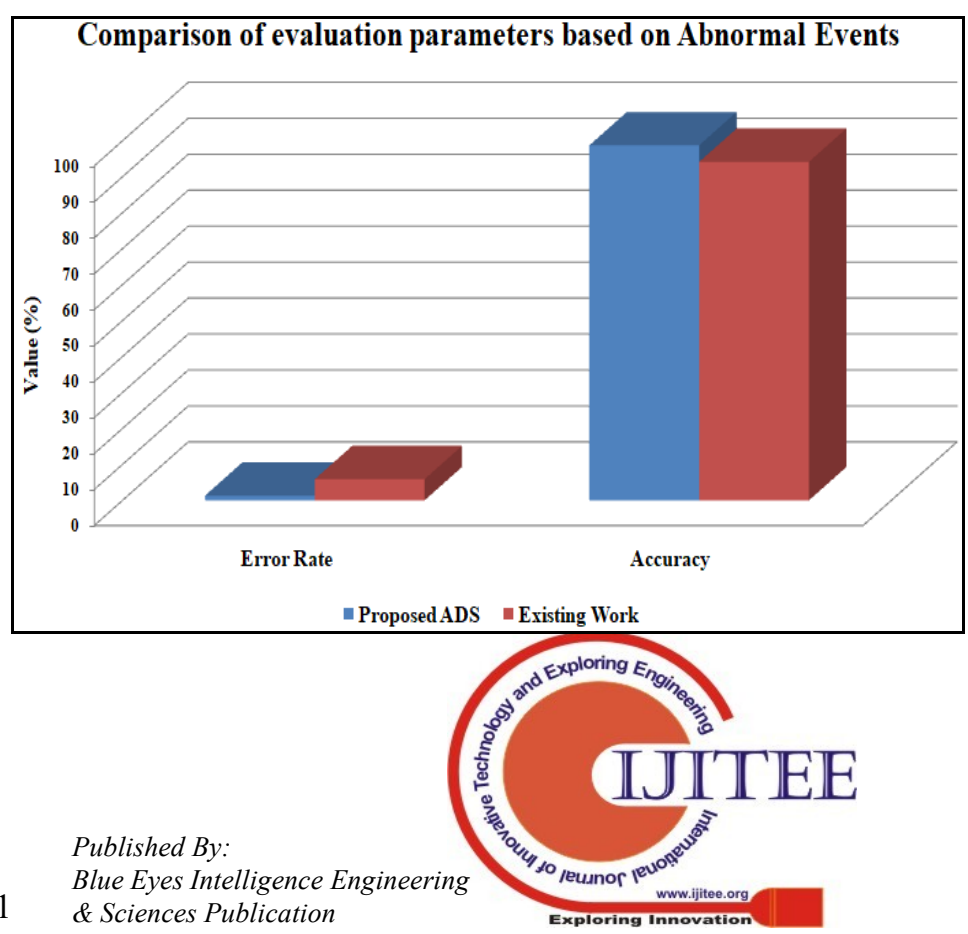




\section{An Optimized CNN based Real World Anomaly Detection in Surveillance Videos}

Figure 9: Comparison of evaluation parameters based on Non-BCC

The comparison of evaluation parameters for proposed and existing work is depicted in figure 8 . For the proposed work on the basis of abnormal event data, average error rate is $1.29 \%$ and average accuracy (AUC) $98.71 \%$. By using the concept of GA with MSER and CNN, the accuracy of proposed work is improved because the average error rate is $5.87 \%$ and average accuracy (AUC) $94.12 \%$ for the existing work. The comparison of proposed work with some other existing work, which is considered in survey of proposed work, is described in below table.

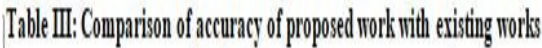

\begin{tabular}{|c|c|}
\hline Authors & Accuracy $(\%)$ \\
\hline |Tagaps Sultani (2018) [I] & 94.59 \\
\hline Suill-Chumg Hso (2018)[2] & 93.1 \\
\hline Hung (2018)/3] & 91.3 \\
\hline Proposed roork & 97.9 \\
\hline
\end{tabular}

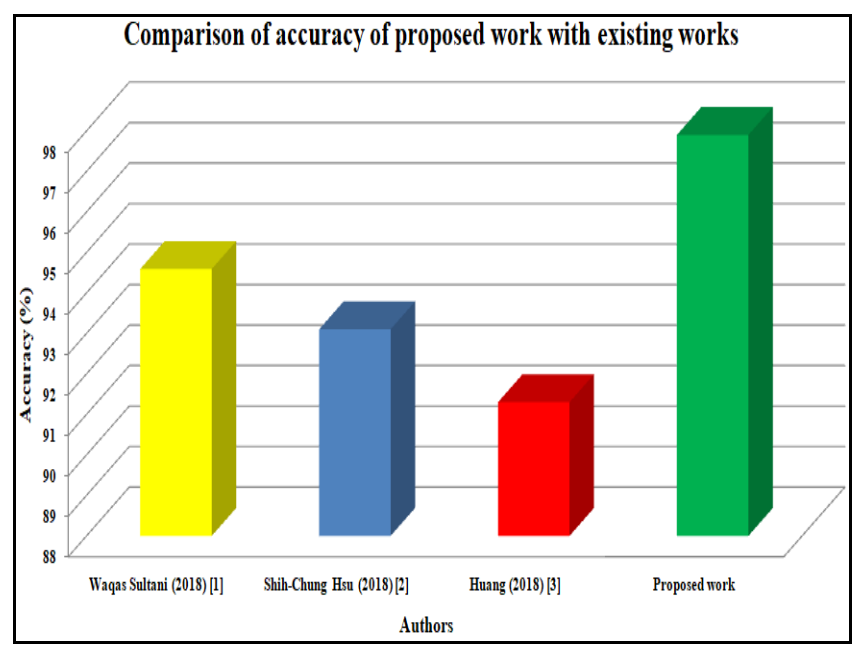

Figure 10: Comparison of accuracy of proposed work with existing works

Figure 10 represents the comparative analysis of existing work based on the classification accuracy. From the figure we observe that the accuracy achieve by proposed work is better than other author by using the hybridization of GA with CNN using MSER feature extraction technique.

\section{Conclusion and Future Work}

In this paper, optimized $\mathrm{CNN}$ based real world anomaly detection in surveillance videos is proposed. It provides a detailed view of the different applications and potential challenges of anomaly detection. The proposed segmentation algorithm is experimented with several surveillances video for detecting and locating abnormal events in the video according to the frames. So, new concept based on the threshold can be used to improve the correctness of temporal segmentation algorithms with the $\mathrm{CNN}$ and GA technique. From the result analysis of proposed and existing work, it seems to be the system accuracy is more than $98 \%$ to detect the anomaly from the surveillance video. To validate the proposed ADS, a new large-scale anomaly dataset consisting of a variety of real world anomalies is introduced which is known as UCF-Crime Dataset. The experimental results on this dataset show that our proposed anomaly detection approach performs significantly better than baseline methods and the accuracy is improved by $3 \%$ as compare to the existing work [1].

In the future work, hybridization artificial intelligence techniques can be used to enhance the performance of proposed anomaly detection from surveillances video. Feature extraction algorithms can also be used to classify the abnormality of events with more accurately so that the efficiency of anomaly detection system can be improved. In addition, the framework can be extended with the objective of anomaly identification, traffic information analysis, traffic prediction, route suggestion, smart park management, etc.

\section{REFERENCES}

1. Sultani, Waqas, Chen Chen, and Mubarak Shah. "Real-world anomaly detection in surveillance videos." Proceedings of the IEEE Conference on Computer Vision and Pattern Recognition. 2018.

2. Hsu, Shih-Chung, et al. video-based abnormal human behavior detection for psychiatric patient monitoring." Advanced Image Technology (IWAIT), 2018 International Workshop on. IEEE, 2018.

3. Huang, Shaonian, Dongjun Huang, and Xinmin Zhou. "Learning Multimodal Deep Representations for Crowd Anomaly Event Detection." Mathematical Problems in Engineering 2018 (2018).

4. Cheng, Kai-Wen, Yie-Tarng Chen, and Wen-Hsien Fang. "Gaussian process regression-based video anomaly detection and localization with hierarchical feature representation." IEEE Transactions on Image Processing 24.12 (2015): 5288-5301

5. Cheng, Kai-Wen, Yie-Tarng Chen, and Wen-Hsien Fang. "Video anomaly detection and localization using hierarchical feature representation and Gaussian process regression." Proceedings of the IEEE Conference on Computer Vision and Pattern Recognition. 2015.

6. Agrawal, Vartika, and Satish Chandra. "Feature selection using Artificial Bee Colony algorithm for medical image classification." Contemporary Computing (IC3), 2015 Eighth International Conference on. IEEE, 2015.

7. Subanya, B., and R. R. Rajalaxmi. "Feature selection using Artificial Bee Colony for cardiovascular disease classification." Electronics and Communication Systems (ICECS), 2014 International Conference on. IEEE, 2014.

8. Uijlings, Jasper RR, et al. "Realtime video classification using dense hof/hog." Proceedings of international conference on multimedia retrieval. ACM, 2014

9. Abu-Mouti, Fahad S., and Mohamed E. El-Hawary. "Overview of Artificial Bee Colony (ABC) algorithm and its applications." Systems Conference (SysCon), 2012 IEEE International. IEEE, 2012.

10. Kharazmi, Pegah, et al. "Automated detection and segmentation of vascular structures of skin lesions seen in Dermoscopy, with an application to basal cell carcinoma classification." IEEE journal of biomedical and health 
informatics 21.6 (2017): 1675-1684.

11. M. J. Khan, A. Zafar and K. S. Hong, "Comparison of brain areas for executed and imagined movements after motor training: An fNIRS study," 2017 10th International Conference on Human System Interactions (HSI), Ulsan, South Korea, 2017, pp. 125-130.

12. R. Majid Mehmood, R. Du and H. J. Lee, "Optimal Feature Selection and Deep Learning Ensembles Method for Emotion Recognition from Human Brain EEG Sensors," in IEEE Access, vol. 5, no. , pp. 14797-14806, 2017.

13. A Minz and C. Mahobiya, "MR Image Classification Using Adaboost for Brain Tumor Type," 2017 IEEE 7th International Advance Computing Conference (IACC), Hyderabad, 2017, pp. 701-705.

14. H. Rao, P. V. Naganjaneyulu and K. S. Prasad, "Brain Tumor Detection and Segmentation Using Conditional Random Field," 2017 IEEE 7th International Advance Computing Conference (IACC), Hyderabad, 2017, pp. 807-810.

15. R. A. Jasmine and P. A. J. Rani, "A two phase segmentation algorithm for MRI brain tumor extraction," 2016 International Conference on Control, Instrumentation, Communication and Computational Technologies (ICCICCT), Kumaracoil, 2016, pp. 437-440.

16. M. Gupta, B. V. V. S. N. P. Rao and V. Rajagopalan, "Brain Tumor Detection in Conventional MR Images Based on Statistical Texture and Morphological Features," 2016 International Conference on Information Technology (ICIT), Bhubaneswar, 2016, pp. 129-133

17. G. Singh and M. A. Ansari, "Efficient detection of brain tumor from MRIs using K-means segmentation and normalized histogram,” 2016 1st India International Conference on Information Processing (IICIP), Delhi, 2016, pp. 1-6.

\section{AUTHORS PROFILE}

Divya Thakur received B.tech Degree in Computer Science \& Engineering from R.I.E.I.T, Ropar and pursuing M.tech from Chandigarh University.Her research areas are DIP, Machine Learning.

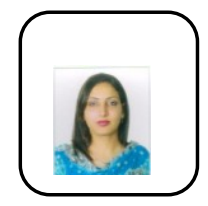

Rajdeep Kaur received M.tech in CSE and pursuing $\mathrm{PhD}$ in Machine Learning and 12 papers published and have ISTE membership. 